\title{
Research Paper: \\ Development of Communicative Functions in Normal Persian-speaking Children from 12 to 18 Months of Age: A Longitudinal Study
}

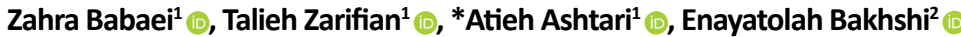

1. Department of Speech Therapy, University of Social Welfare and Rehabilitations Sciences, Tehran, Iran

2. Department of Biostatistics and Epidemiology, Faculty of Rehabilitation, University of Social Welfare and Rehabilitations Sciences, Tehran, Iran.

\begin{tabular}{|c|c|}
\hline $\begin{array}{l}\text { Use your device to scan } \\
\text { and read the article online }\end{array}$ & Cftation Babaei Z, Zarifian T, Ashtari A, Bakhshi E. [Development of Communicative Functions in Normal Persian-speaking \\
\hline ata & $\begin{array}{l}\text { Children from } 12 \text { to } 18 \text { Months of Age: A Longitudinal Study (Persian)]. Archives of Rehabilitation. 2020; 21(2):220-235. https:// } \\
\text { doi.org/10.32598/RJ.21.2.2956.1 }\end{array}$ \\
\hline aris & 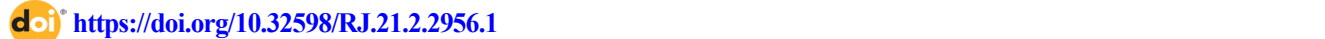 \\
\hline
\end{tabular}

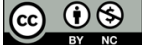

Received: 06 Jan 2019 Accepted: 09 Dec 2019 Available Online: $01 \mathrm{Jul} 2020$

Keywords:

Communicative function, Behavior regulation, Social interaction, Joint attention, Typically developing children

\begin{abstract}
Objective Communication can affect the personal and social life of people. According to International Classification of Functioning, Disability and Health (ICF), communication is one of the most important components of social participation and can have different functions such as behavior regulation (regulating the behavior of others to achieve a particular goal), social interaction (drawing attention of others to the self) and joint attention (attracting the attention of others to an event or particular object). Having knowledge of the development of communicative functions is so important in assessment, diagnosis, prognosis, and early intervention. Despite the importance and effect of communicative functions in language and communication development, no study has been conducted yet that examines the communicative function development of normal Persian-speaking toddlers. The purpose of this study was to investigate the development of communicative functions in normal Persian-speaking children from 12 to 18 months of age, regardless of communicative expressions (such as gestures, vocalization, and words).

Materials \& Methods This is a longitudinal observational study. The communicative function of 11 monolingual Persian-speaking 12-month-old children (7 boys and 4 girls) living in Tehran, Iran was investigated for 7 months. Sampling was done by using purposive and snowball non-probability sampling methods. All children had healthy medical history and normal growth, were from a family with a moderate socioeconomic status, and their parents had at least a high school diploma. The participants' developmental status was assessed by Ages and Stages Questionnaire. A demographic form with acceptable content validity was also used to determine their demographic characteristics. For 7 months (once a month, each session for one hour), the examiner filmed the childmother interaction during a semi-structured play with a set of toys at the child's home. Afterwards, the recorded videos were coded according by the researcher and then entered into the SPSS V. 22 software. Descriptive statistics including mean and standard deviation were used to describe the data. The Shapiro-Wilk test was used to determine the normality of data distribution, and repeated measures ANOVA (within-subject comparison) was used to analyze the data. Two raters were taught about the coding and rating of the communicative functions. To verify the validity of the data coding, $20 \%$ of the recorded samples were given to them to code communicative functions. The coding validity was determined by calculating the Intra-class Correlation Coefficient (ICC).

Results The mean frequency of behavior regulation and social interaction was constant from 12 to 18 months of age $(P<0.05)$, while the mean frequency of joint attention was constant from 12 to 15 months of age $(P<0.05)$ and then significantly increased at months $16(P=0.019), 17(P=0.023)$ and $18(P=0.003)$ compared to the 12 th month. The ICC value as the criterion for coding validity was reported $90 \%$.

Conclusion The mean frequency of joint attention increased significantly from 12 to 18 months of age, while it was constant for behavior regulation and social interaction. Our findings are consistent with the ICF's social participation, stating that a child uses a particular type of communicative function to increase its participation in the society.
\end{abstract}

* Corresponding Author:

Atieh Ashtari

Address: Department of Speech Therapy, University of Social Welfare and Rehabilitations Sciences, Tehran, Iran.

Tel: +98 (21) 22180043

E-Mail: atieh.ashtarislp@gmail.com 


\section{Extended Abstract}

\section{Introduction}

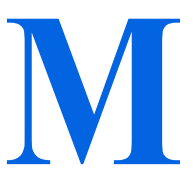

an is a social being who instinctively has a great desire to communicate with others from childhood. Communication is one of the important components of social participation and can be done for different purposes. In International Classification Of Functioning, Disability and Health (ICF), communication is one of the important dimensions of social participation [1-4]. According to speech act theory, verbal communication is formed during the pre-linguistic period in three stages: preverbal, incomplete verbal and verbal [5]. Except in the first stage, the child's communication in the next stages is purposeful $[3,6]$ and is accompanied by various communication functions, including regulating the behavior of others (the child's effort to ask others and regulates the behavior of others by using hands), social interaction (the child's attempt to draw attention to herself/himself) and joint attention (the child's attempt to draw attention to a particular event or object) [2, 7-9]. There are different opinions regarding the growth and the frequency of communicative functions; however, there is general agreement among researchers that the communicative functions of behavior regulation and social interaction occur earlier than the joint attention, at the age of about 9 months [7, 10, 11].

Some other studies have shown that the communicative functions of behavior regulation and joint attention occur at the end of the first year of a child's life [12]. According to studies, in the second year of the child's life, the frequency of joint attention is higher than the other two communicative functions [1]. Having knowledge of the growth trend of communicative functions is effective in evaluating, diagnosing, predicting and timely intervention [13-18]. Any change in the development pattern of communicative functions can be a caution for possible communication problems, and as a result, more measures should be taken for timely diagnosis and intervention [1, 19, 20]. Communicative functions in children with different types of communication and language disorders are not only different from each other but also different from peers. In fact, the frequency of communicative functions can be an important indicator for early diagnosis of communication and language disorders. Thus, the lack of proper development of communicative functions and changing their development pattern can predict the existence of possible communication and language problems in the future [2].

None of the existing studies in this field have directly examined the development trend of communicative functions, and they are not even up-to-date; so there is a research gap in this field. Moreover, due to cultural differences [21], the findings of studies in other countries cannot be generalized to Iranian society; therefore, it is necessary to study the communicative functions in an area according to its culture. The aim of this study was to investigate the development of communicative functions in Persian-speaking children with a normal growth from 12 to 18 months of age and to find quantitative scales for these functions that children use in the prelinguistic period.

\section{Materials \& Methods}

In this longitudinal/observational study, the communicative function of 11 monolingual Persian-speaking 12-month-old children ( 7 boys and 4 girls) was examined for 7 months (up to the age of 18 months). The sample size was selected with $80 \%$ statistical power. This age group was selected because the onset of communicative functions following intentional communication is from the incomplete verbal communication stage (12-18 months). The samples were selected first by purposive sampling method and then by snowball sampling method. All children had a history of healthy medicine, normal development, parents with at least a high school diploma, and came from came from middle-class families in terms of socio-economic status living in Tehran [22]. Data collection tools were the Ages \& Stages Questionnaire (ASQ), a demographic form, a video recorder (HDR-XR100, Sony, Japan), and set of toys including bubble makers, balloons, polished dolls, plastic animals, tableware, sanitary ware, books and peekaboo game.

The ASQ was first used to check the status of the development of children. Standardization and validation of its Persian version was performed by Sajedi et al. [23]. They reported a Cronbach's alpha coefficient of 0.79 in total, and the construct validity of the questionnaires was confirmed by factor analysis. The demographic form was used to survey age, gender, birth order, mother's level of education, socio-economic status of the family, the presence or absence of bilingualism, and a history of speech, language and communication disorders. Its content validity was evaluated qualitatively and the questions were modified based on the opinions of 14 speech and language pathologists and then provided to the parents of the children. After obtaining informed consent from the parents, the examiner went to the children's home once a month for 7 months, and filmed for 1 hour of parent-child interaction during a semistructured play with toys (15 minutes of free play with the child's own toys and 45 minutes of structured play with the designed toys). 
The researcher then observed each of the recorded videos and prepared a chronological report of them. The obtained data were encrypted and then entered into SPSS V. 22 software. All comparisons were considered significant at $\mathrm{P}<$ 0.05. Descriptive statistics including mean and standard deviation were used to describe the data. To measure the normality of the data distribution, the Shapiro-Wilk test was performed, and for data analysis, repeated measures ANOVA and binary comparison were used. In order to evaluate the validity of data coding, after teaching data coding to raters who were expert in the field of communicative function development, $20 \%$ of the recorded behavioral samples were given to two of them to independently code the data. The coding validity of the recorded observations was also determined by the Intraclass Correlation Coefficient (ICC).

\section{Results}

In this section, the findings related to communicative functions were considered from two perspectives: 1 . Comparison of communicative function development in two consecutive months; and 2. Comparison of communicative function development in the 12th month with the development in other months. According to the results in Table 1 and Figure 1, the most frequent communication function developed from the first 12 to 18 months is joint attention followed by behavior regulation and social interaction. According to the results in Table 2, repeated measures ANOVA results indicated that there was no significant difference between the mean frequency of behavior regulation and social interaction in two consecutive months $(\mathrm{P}>0.05)$. More- over, the ANOVA results showed that the frequency of joint attention was not significantly different from 13 to 14,14 to 15,16 to 17 , and 17 to 18 months $(\mathrm{P}>0.05)$, but it was significant from the month 12 to $13(\mathrm{P}=0.028)$ and from 15 to $16(\mathrm{P}=0.046)$. According to the ANCOVA results in Table 3 , there was no significant difference between the mean frequency of behavior regulation and social interaction at $13,14,15,16,17$ and 18 months and their frequency at 12 months $(\mathrm{P}>0.05)$. Furthermore, there was no significant difference between the mean frequency of joint attention at 3 , 14,15 months and its frequency at 12 months $(\mathrm{P}>0.05)$, but the difference significant between its frequency at 16,17 , and 18 months and at 12 month was significant $(\mathrm{P}<0.05)$. Moreover, after coding of $20 \%$ of the recorded videos by the raters, the value of the ICC which was used as the criterion of coding validity was obtained $90 \%$.

\section{Discussion}

In this longitudinal observational study, the communicative functions of 11 Persian-speaking monolingual children ( 7 boys and 4 girls) living in Tehran were examined while interacting with their mothers. The results of the study showed that the mean frequency of two communicative functions of behavior regulation and social interaction remained constant from the age of 12 to 18 months, while the frequency of joint attention, despite remaining constant from the age of 12 to 15 months, increased at the age of 16,17 , and 18 months compared to its status at the age of 12 months. The comparison of the frequency of communicative functions in each month showed that the joint at-

Table 1. Mean \pm SD of the frequency of communicative functions in children from the age of 12 to 18 months

\begin{tabular}{cccc}
\hline & \multicolumn{3}{c}{ Mean \pm SD } \\
\cline { 2 - 4 } Communicative Functions & $\mathbf{1 2}$ Months & $\mathbf{1 3}$ Months & 14 Months \\
\hline behavior regulation & $57.636 \pm 16.001$ & $54.090 \pm 22.536$ & $59.272 \pm 20.115$ \\
Social interaction & $29.545 \pm 13.574$ & $29.272 \pm 20.253$ & $33.818 \pm 22.824$ \\
Joint attention & $83.363 \pm 44.238$ & $104.000 \pm 50.025$ & $108.909 \pm 52.658$ \\
\hline
\end{tabular}

\begin{tabular}{ccccc}
\hline \multirow{2}{*}{$\begin{array}{c}\text { Communicative Func- } \\
\text { tions }\end{array}$} & \multicolumn{4}{c}{ Mean \pm SD } \\
\cline { 2 - 5 } & 15 Months & 16 Months & 17 Months & 18 Months \\
\hline behavior regulation & $64.181 \pm 25.697$ & $65.363 \pm 29.038$ & $62.545 \pm 25.959$ & $68.727 \pm 20.833$ \\
Social interaction & $34.181 \pm 20.158$ & $43.272 \pm 32.205$ & $40.090 \pm 31.315$ & $44.181 \pm 29.552$ \\
\hline Joint attention & $104.636 \pm 58.435$ & $135.909 \pm 51.773$ & $128.000 \pm 40.916$ & $156.362 \pm 54.759$ \\
\hline
\end{tabular}


Table 2. Comparing the development of communicative functions in two consecutive months

\begin{tabular}{|c|c|c|c|c|}
\hline \multicolumn{2}{|c|}{ communicative functions } & Mean & $\mathbf{F}$ & Sig. \\
\hline \multirow{6}{*}{ Behavior regulation } & $12-13$ & 138.273 & 0.522 & 0.487 \\
\hline & $13-14$ & 295.364 & 0.475 & 0.506 \\
\hline & $14-15$ & 265.091 & 0.940 & 0.355 \\
\hline & $15-16$ & 15.364 & 0.035 & 0.858 \\
\hline & $16-17$ & 87.364 & 0.125 & 0.731 \\
\hline & $17-18$ & 420.364 & 0.629 & 0.446 \\
\hline \multirow{6}{*}{ Social interaction } & $12-13$ & 0.818 & 0.002 & 0.963 \\
\hline & $13-14$ & 277.273 & 1.586 & 0.236 \\
\hline & $14-15$ & 1.455 & 0.009 & 0.928 \\
\hline & $15-16$ & 909.091 & 2.887 & 0.120 \\
\hline & $16-17$ & 111.364 & 0.252 & 0.626 \\
\hline & $17-18$ & 184.091 & 0.152 & 0.705 \\
\hline \multirow{6}{*}{ Joint attention } & $12-13$ & 4684.455 & 6.546 & $0.028^{*}$ \\
\hline & $13-14$ & 184.091 & 0.618 & 0.450 \\
\hline & $14-15$ & 131.273 & 0.159 & 0.699 \\
\hline & $15-16$ & 10757.818 & 5.207 & $0.046 *$ \\
\hline & $16-17$ & 633.091 & 1.066 & 0.326 \\
\hline & $17-18$ & 8849.455 & 4.200 & 0.068 \\
\hline
\end{tabular}

$*(\mathrm{P}<0.05)$ is significant

Rehabilitation

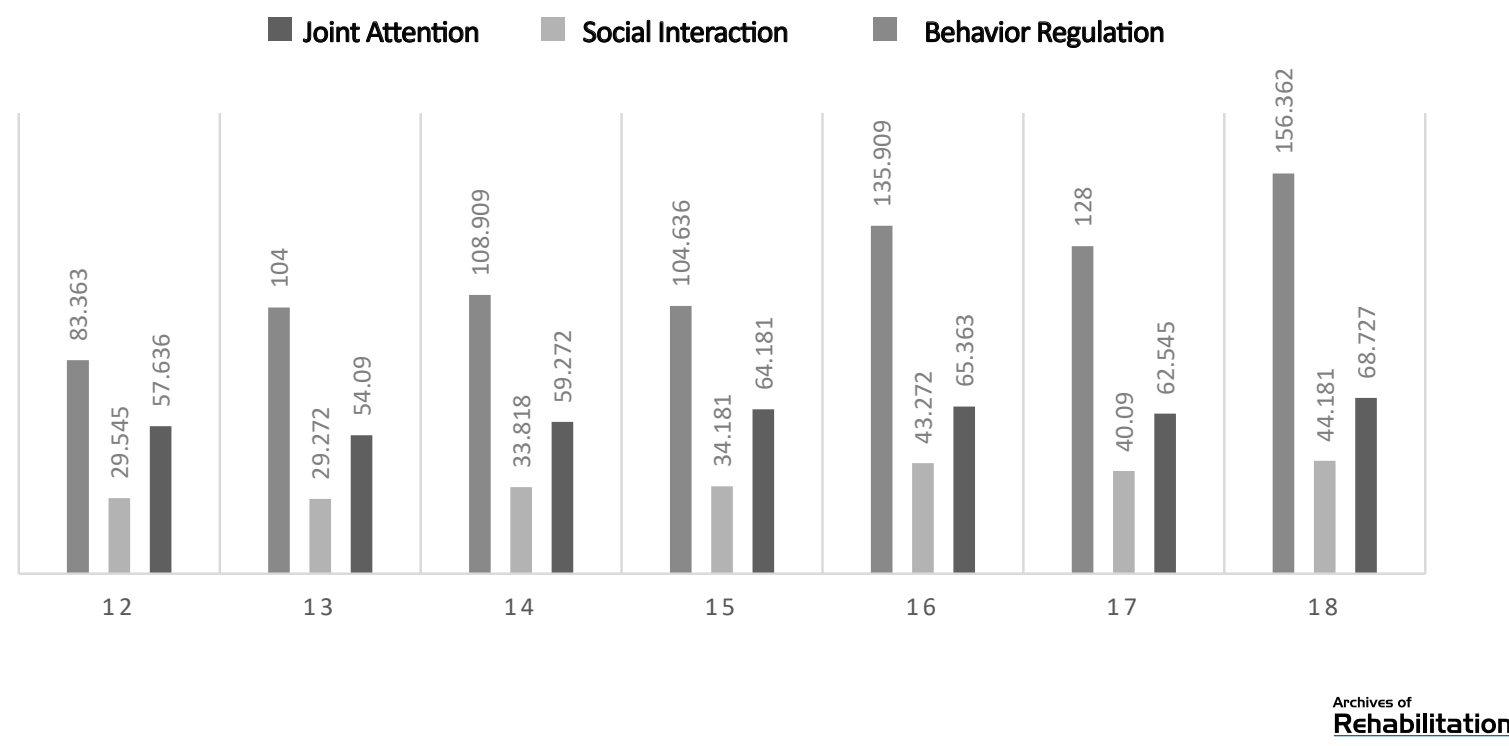

Figure 1. Comparing the mean frequency of communicative functions in children from the age of 12 to 18 months 
Table 3. Comparing the development of communicative functions in the 12th month with the development in other months

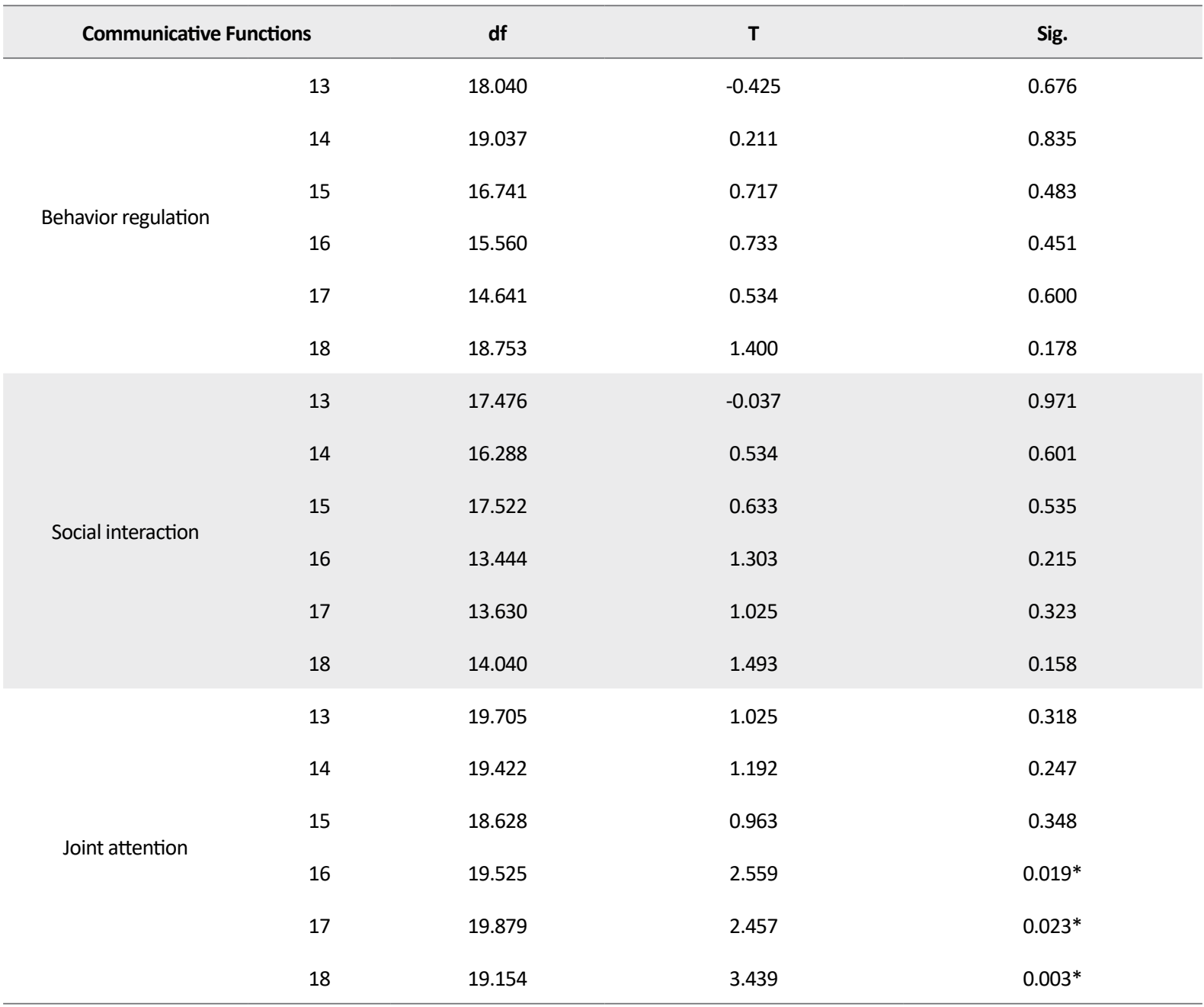

$*(\mathrm{P}<0.05)$ is significant

tention had the highest frequency compared to other two functions of behavior regulation and social interaction. Few studies have evaluated the development of communicative functions; however, the results of this study are consistent with the findings of Carpenter et al. on the communicative functions from the age of 8 months [11], because in the age range of 12 to 18 months, the frequency and development of gestures are more important than the age of onset. The stability of the frequency of behavior regulation and social interaction from 12 to 18 months of age may due to the research method and conditions, and that the play and context were designed to require the same performance of these two functions over 7 months [24]. This is consistent with the theory of socio-behavioral learning based on the effect of the child's upbringing and living environment or play environment conditions on the child's performance [25, 26 ] and the theory of environment-child interaction. A child interacts with others based on communicative goals appro-

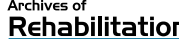

priate to the environment where s/he presents [27]; if $\mathrm{s} / \mathrm{he}$ failed to communicate appropriately according to the above conditions, s/he may have problems with social participation and her/his communicative goals may be ineffective. This may cause the child's interactive partner not to pay enough attention to the child's communication due to the lack of clarity in the child's communicative functions. As a result, the child may have limitations in social relations [4].

Stability of the frequency of joint attention from 12 to 15 months of age and its upward growth at 16,17 and 18 months of age compared to that at 12 months of age may be associated with increased ability of the child to create a interaction triangle (a communication triangle between child, objects, events or interactive partner). On the other hand, increasing the children's skills to produce pointing gesture [28], as an important way to perform joint attention, can be effective in increasing the frequency of joint attention [18]. This finding is consistent with the social/behavioral learn- 
ing theory based on the imitation of observations [25, 26], the theory of cognitive development indicating the formation of child cognition within the society [29], the theory of social interaction indicating learning in social contexts [30] and the theory of ecological systems [27]. According to these theories, by observing the behavior of mothers and other people in society and imitating the behaviors that they use to draw each other's attention to the environment, tools and people around them, the child learns the communicative function of joint attention and uses it more. The frequency of behavior regulation and social interaction was remained constant from 12 to 18 months of age, but the frequency of joint attention increased from 12 to 18 months of age. These findings are consistent with the social participation dimension of ICF; the child uses a specific communicative function to increase his/her participation in society as required by the environment.

Another result of this study is related to the comparison of the frequencies of communication functions in each month. These results showed that the joint attention had the highest frequency compared to other two communicative functions, which was consistent with the results of Topbas et al. [37]. Due to higher cognitive abilities and motor skills in the age range of 12-18 months, the child prefers to do the activities herself/himself; as a result, s/he uses the behavior regulation function less than the joint attention. On the other hand, the context of the play is such that the use of social interaction function is limited to a few situations and the child engages in social interaction based on these few situations. But, there are several stimuli (toys and other devices in the room) to share with others and develop the function of joint attention; so it seems logical that the child uses this function more than other communicative functions.

The degree of correlation between raters was determined $90 \%$ by calculating ICC. This result indicates a high agreement among evaluators and probably shows that researchers have provided accurate definitions of the variables and that the coding guidelines of this study are applicable for future studies.

\section{Conclusion}

To our knowledge, the present study is the first longitudinal study to determine the development of communicative functions in normal Persian-speaking children. Examining the development of these functions in the clinical field is very important. This study helps to evaluate, diagnose, predict and intervene in children with communication disorders by using checklists and growth charts. Families, as the first communication partners, and home as the first communication context of the children, are very important for the development of their communicative skills and, therefore, will play an important role in prognosis and effectiveness of interventions for them. Due to the small sample size of the present study, there is a need to be cautious in generalizations of the results. It is suggested that more studies be conducted on the development of communicative function using a larger sample size and considering communication techniques (gestures, vocalization, words, and sentences). Moreover, further studies should be conducted on the role of communicative functions in timely diagnosis of communication and language problems in children.

\section{Ethical Considerations}

\section{Compliance with ethical guidelines}

The present study was approved by the ethics committee of the University of Social Welfare and Rehabilitation Sciences (Code IR.URWR.REC:4931.533).

\section{Funding}

The present paper was extracted from the master MSc. of first author Department of Speech Therapy, University of Social Welfare and Rehabilitations Sciences, Tehran, Iran.

\section{Authors' contributions}

Conceptualization, methodology: Zahra Babaei, Talieh Zarifian, Atieh Ashtari and Enayatullah Bakhshi; Research, drafting: Zahra Babaei; Editing and finalizing the writing, funding, sources: Talieh Zarifian, Atieh Ashtari, Zahra Babaei; Project supervision and management: Talieh Zarifian, Atieh Ashtari, Enayatullah Bakhshi.

\section{Conflicts of interest}

The authors declared no conflict of interest. 
This Page Intentionally Left Blank 


\title{
مقاله بثزوهشى

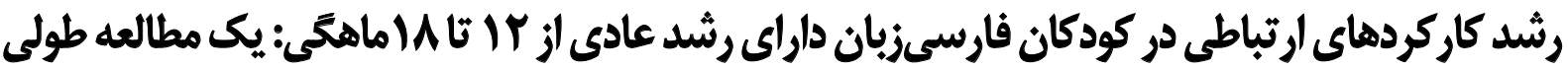

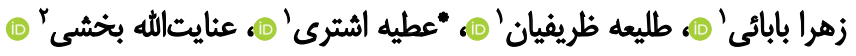 \\ ا. ا.كروه كفتاردرمانى، دانشكده توانيخشى، دانشكاه علوم بهزيستى وتوانبخشى، تهران، ايران.

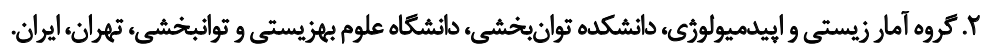

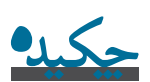

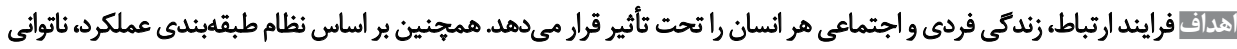

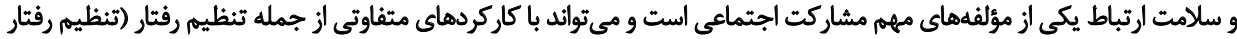

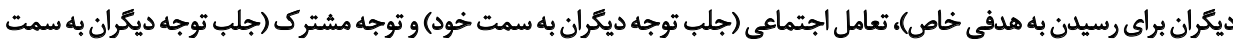

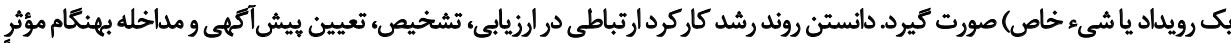

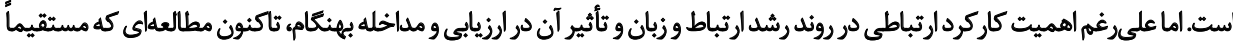

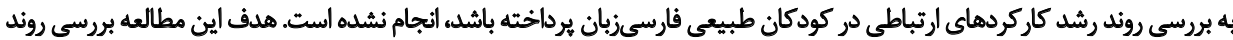

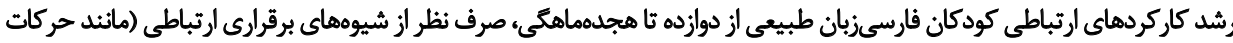
بيائكر، صداسازى و كلمات ) بوده.

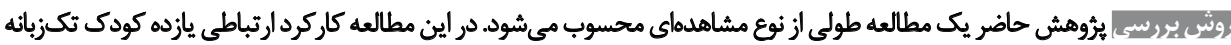

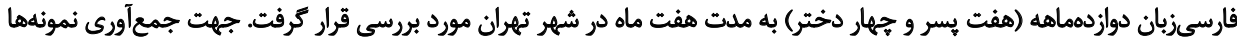

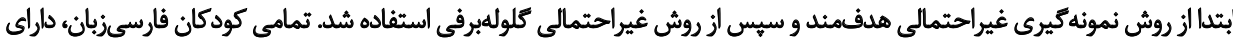

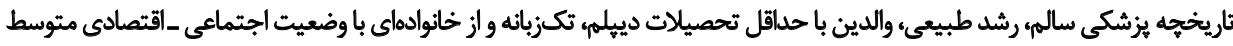

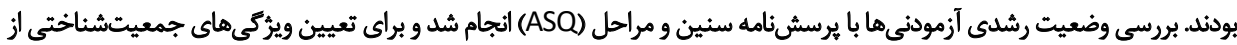

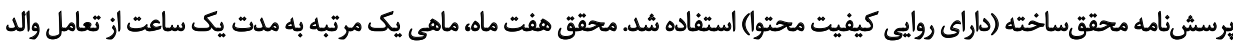

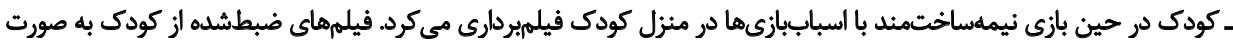

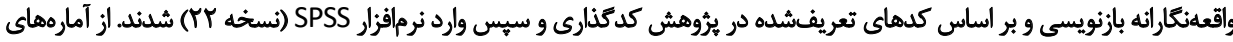

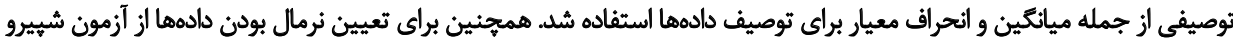

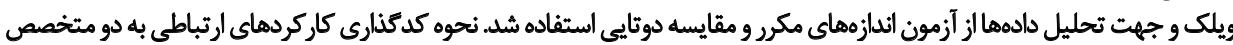

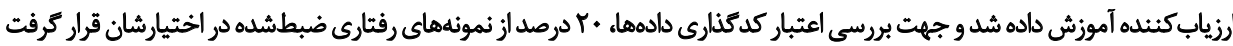

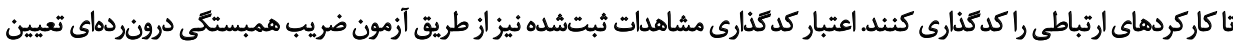

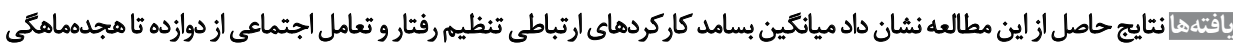

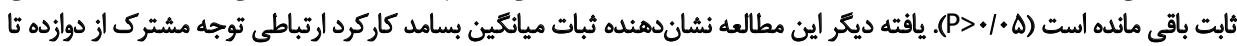

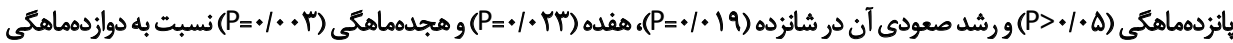

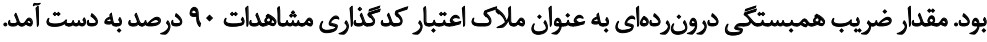

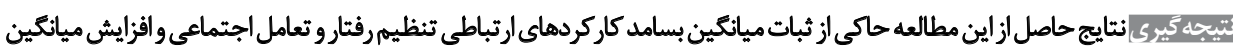

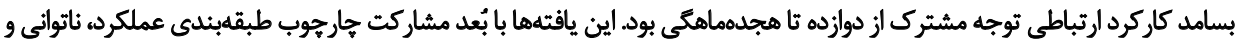

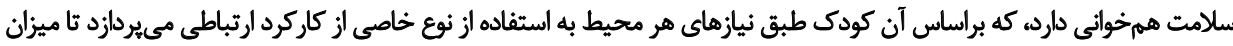
مشاركتش در محيط را افزايش دهد برد

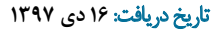

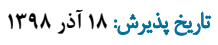

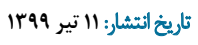

كليدوارٔهها:

كاركرد ارتباطى، تنظيم

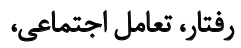
توجه مشترك، كودكان داراى رشد عادى 
مطالعات نيز نشان دادنداند كاركرد ارتباطى تنظيم رفتار و توجه

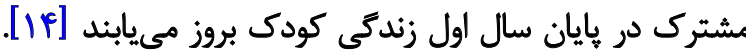

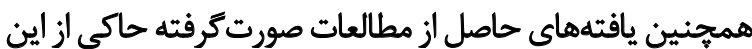

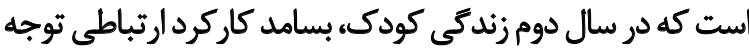

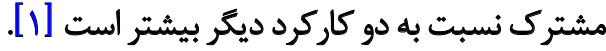
دانستن روند رشد كاركرد ارتباطى در ارزيابي، تشخيص، تعيين

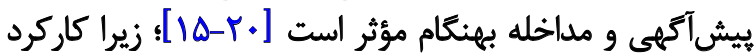

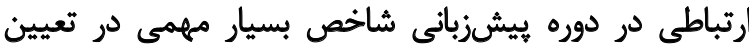

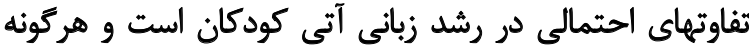

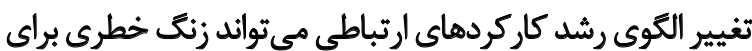

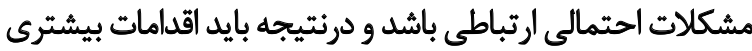

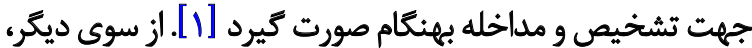

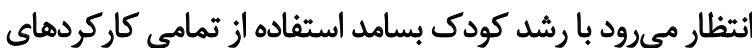

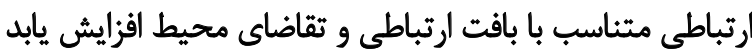

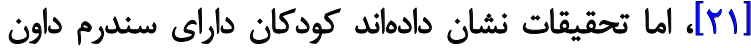

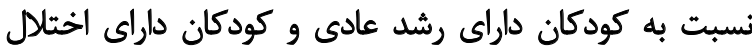

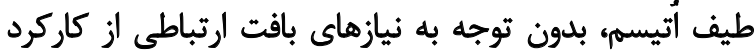

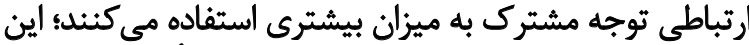

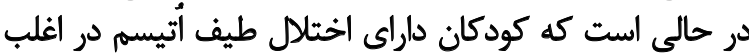

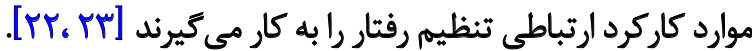

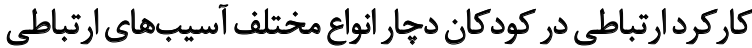

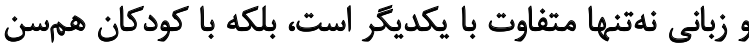

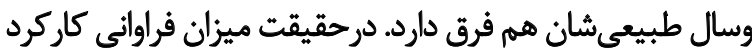

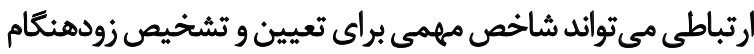

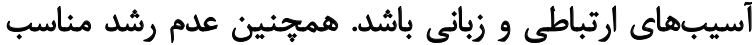

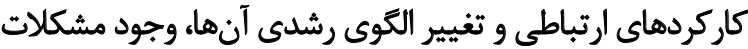

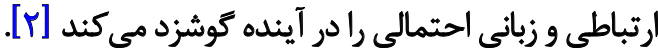

على زغم اهميت كاركردهاى ارتباطى در روند رشد ارتباط و

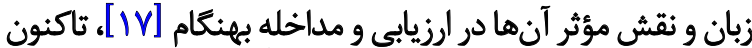

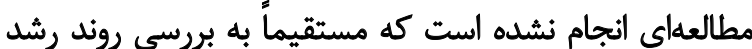

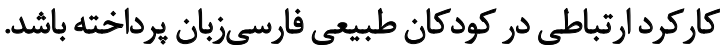

در زمينه كاركردهاى ارتباطى، مطالعات متعددى در جهان

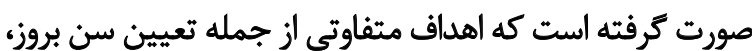

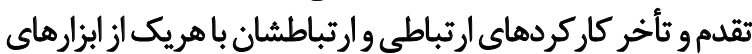

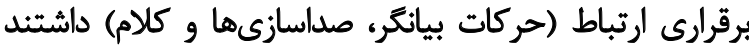

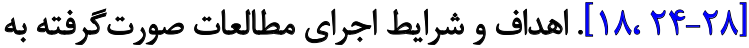

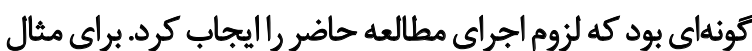

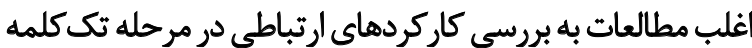

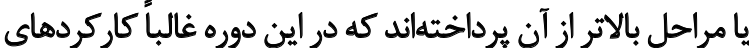

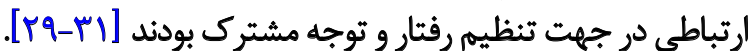

در ادامه به مهمترين مطالعات انجامشده در زمينه كاركردهاى

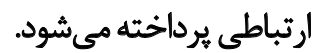

مقدمه

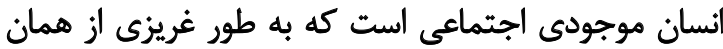

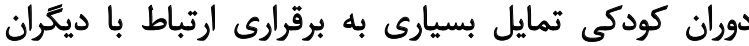

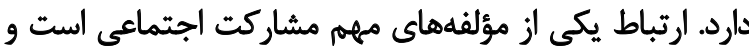

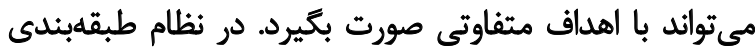

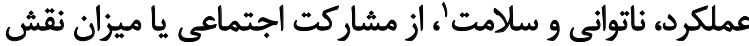

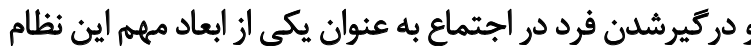

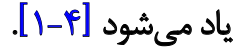
در نظريه كنش كَفتار '، طى دوره بهيشزبانى بروز ارتباط در سه

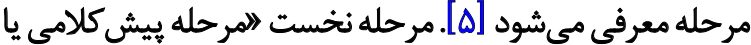

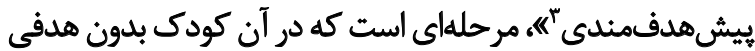

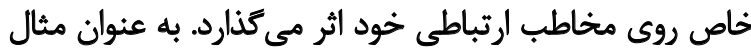

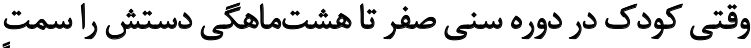

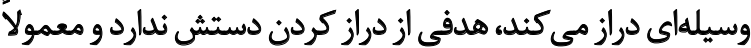

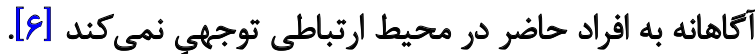

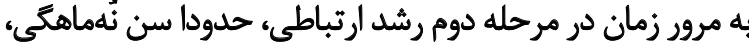

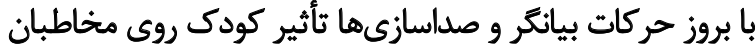

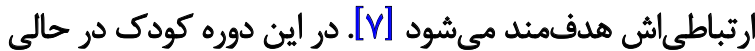

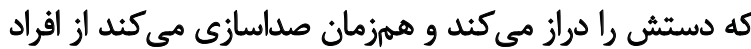

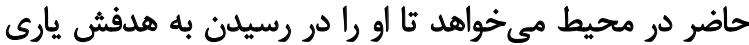

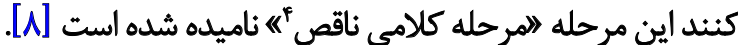
ازز حدود سن سيزدهماهكى كودى وارد مرحله سوم رشد

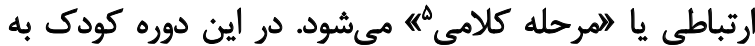

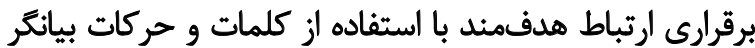

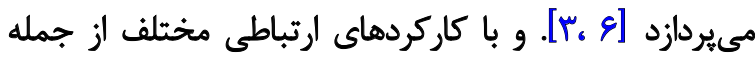

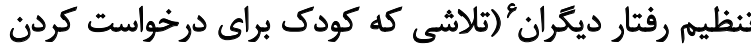

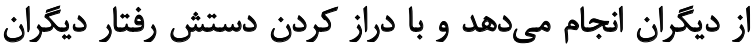

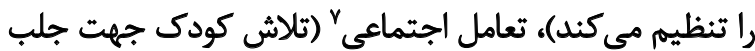

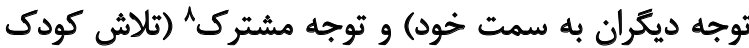

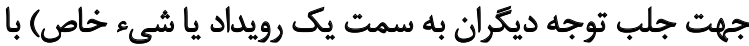

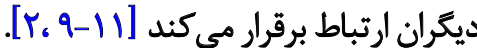

در رابطه با توالى روند رشد و ميزان فراوانى كاركرد ارتباطى توري

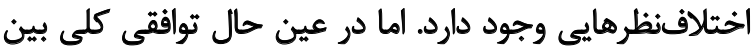

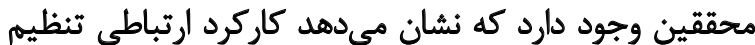

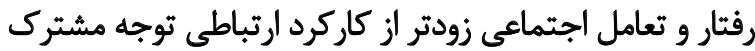

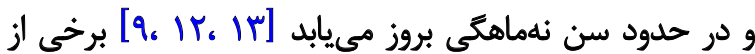

1. International Classification of Functioning, Disability and Health; ICF 2. Speech Act Theory

3. Perlocutionary

4. Illocutionary

5. locutionary

6. Behavior regulation

7. Social interaction

8. Joint attention 
كودى تكز بانه فارسىزبان دوازدمهاهه (هفت يسر و هجهار دختر)

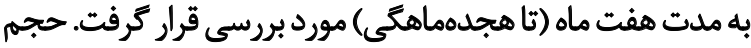

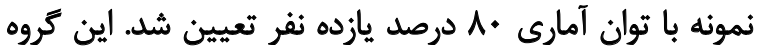

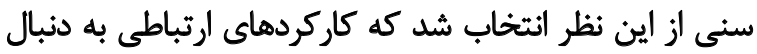

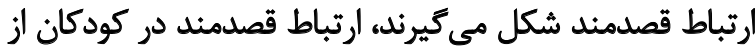

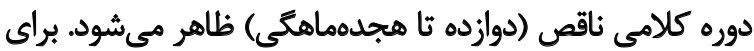

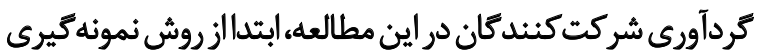

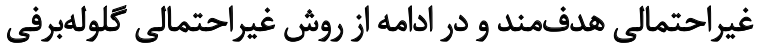

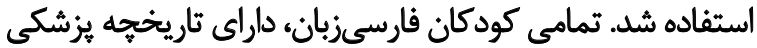

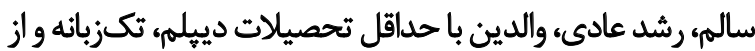

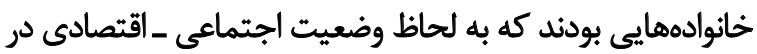
طبقه متوسط كلانشهر تهران [اهب] جاى مي جركرفتند.

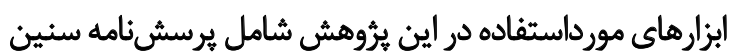

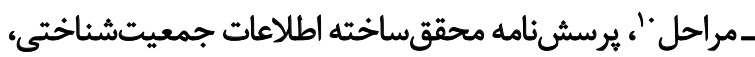

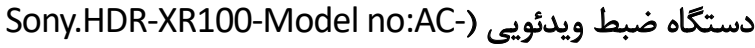

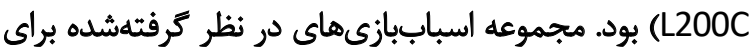

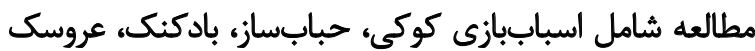

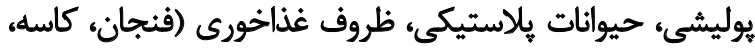

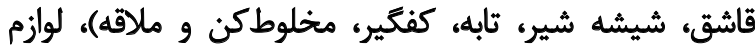

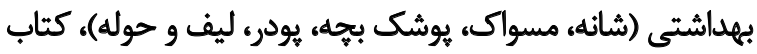

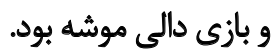

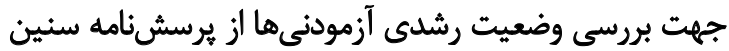

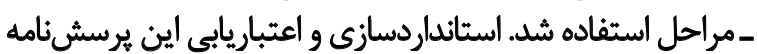

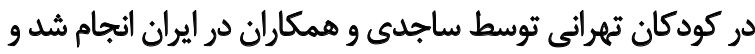

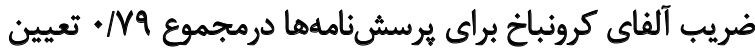

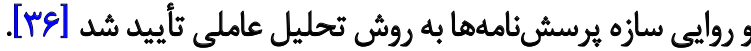

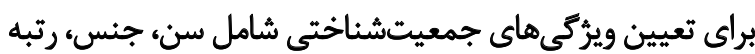

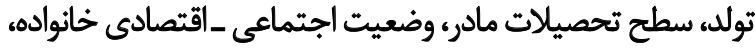

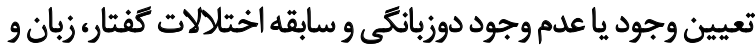

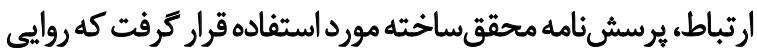

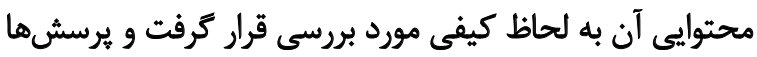

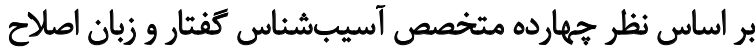

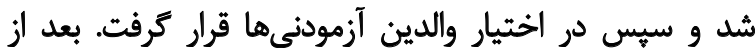

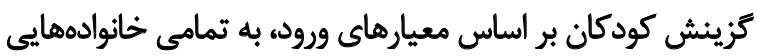

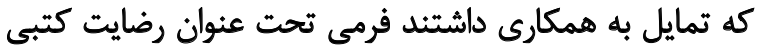

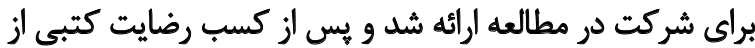

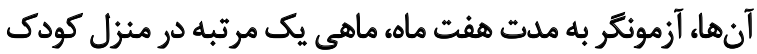

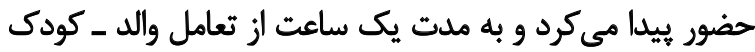

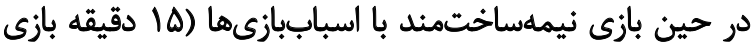

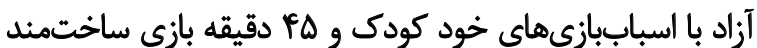

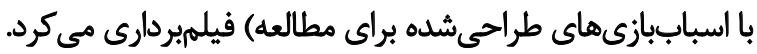

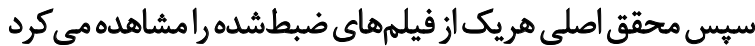

مطالعه كارينتر و كوكيتز روى شش كودى نشان داد كودكان

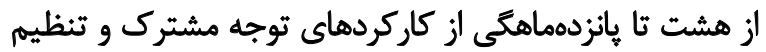

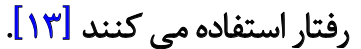

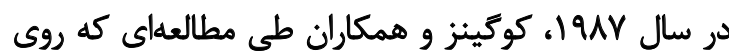

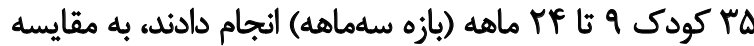

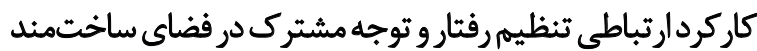

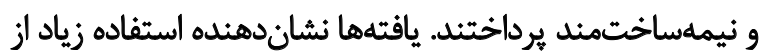

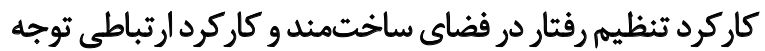

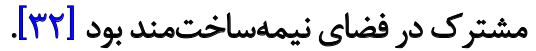

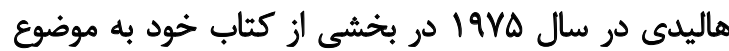

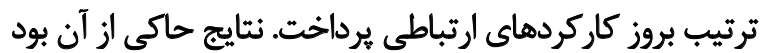

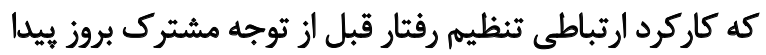

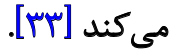

در ايران نيز عليمرادى و همكاران طي مطالعهاي روى بنُه كودى

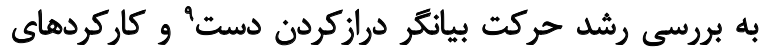

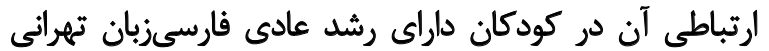

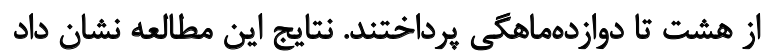

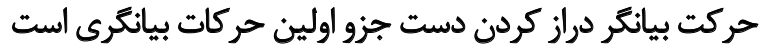

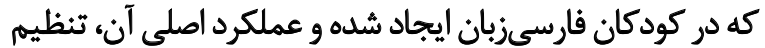

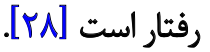

اما همانطور كه در بالا نيز ذكر شد هيجيك از مطالعات

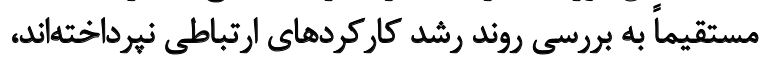

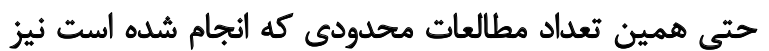

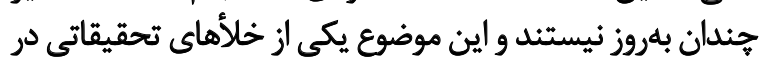

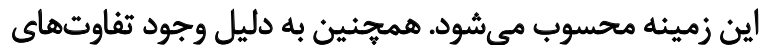

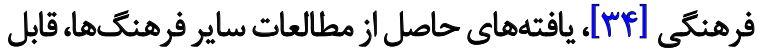

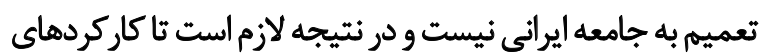

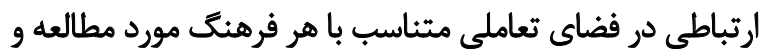
برسى قرار كيرد.

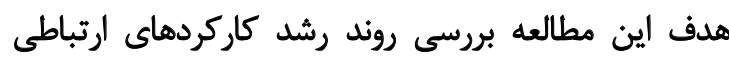

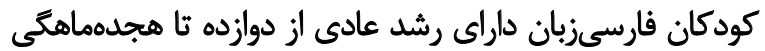

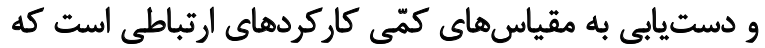

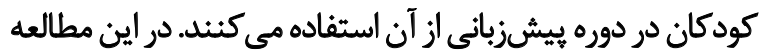

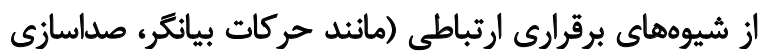

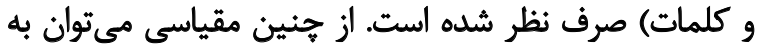

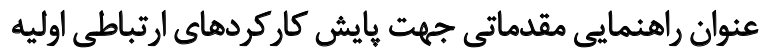

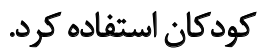
ووشى بروري در اين مطالعه طولى از نوع مشاهدهاي، كاركرد ارتباطى يازده 
ارتباطى از دو منظر مورد توجه قرار كرفت: يكى مقايسه

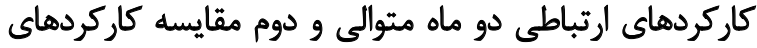

ارتباطى نسبت به ماه دوازدهم.

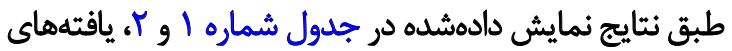

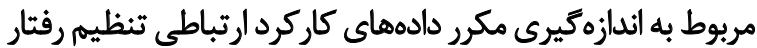

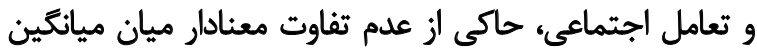

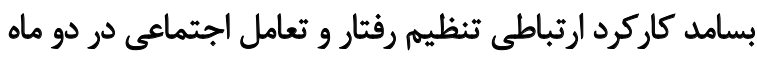

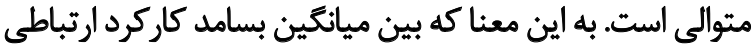

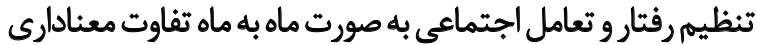

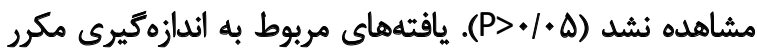

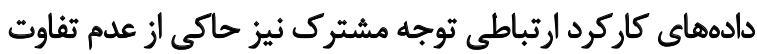

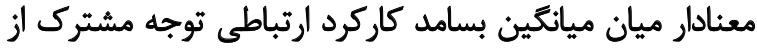

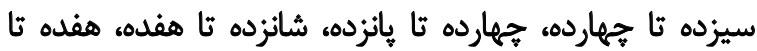

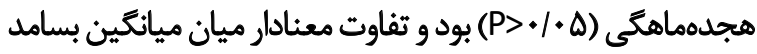

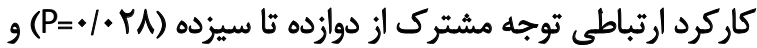

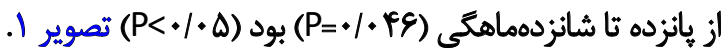
علاوه بر اين، طبق نتايج ذكرشده در جدول شماره با، يافتههاى

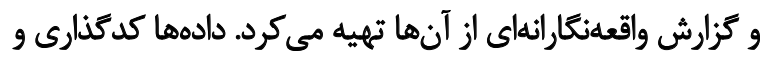

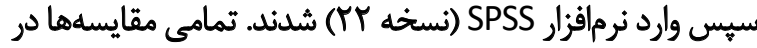
سطح ه ٪ • معنادار در نظر ترفته شد.

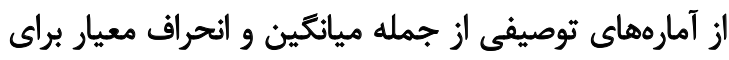

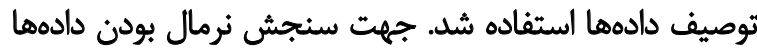

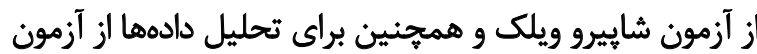

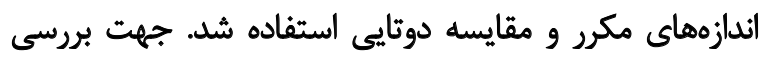

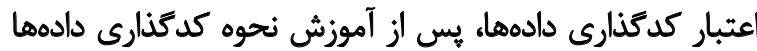

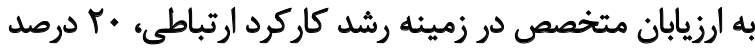

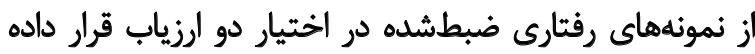

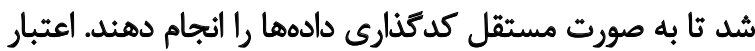

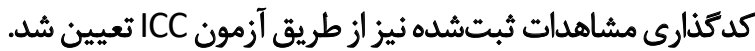

يافتههl

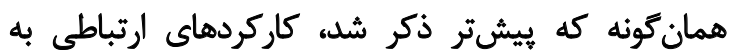

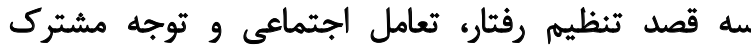

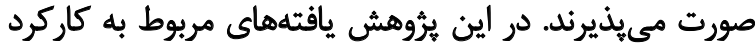

جدول ا. ميانكين بسامد كاركرد ارتباطى در كودكان طبيعى فارسىزبان از با تا ه اماهكى

\begin{tabular}{|c|c|c|c|c|c|c|c|}
\hline 1/ ماهكى & VIاهكى & 7 اماهكى & (ماهكيى & ع (ماهكى & "זاماهكمى & rا ماهكمى & it \\
\hline ENATY & ET/AFA & gA/ReT & Ex/MI & $\Delta Q / T M$ & $\Delta \% \%$. & av/gre & ميانكين بسامد تنظيمه رفتثار \\
\hline$r \cdot / A \pi r$ & $r \Delta / q \Delta q$ & $r q \cdot r \wedge$ & ro/FaV & $r+/ 110$ & Tr/ars & $|8| . .1$ & (انحراف معيار) \\
\hline 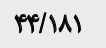 & $p+10 q$. & $R T / T r$ & $m /(M)$ & M/AIA & ra/TM & ravara & ميانكين بساهد تعامل \\
\hline YQ/AOY & rimia & $\pi / T+\Delta$ & $r+/ 1 Q 1$ & MT/AME & $r \cdot /$ ror & $1 w / \Delta n^{e}$ & اجتماعى (انحراف معيار) \\
\hline IDF/MET & $i r N_{++*}$ & $17 \Phi / 9.9$ & 1.tpors & $1+N 9.9$ & $1 . \% \%$ & AT/KR & مياثكين بسامل توجه \\
\hline$\Delta F / N Q q$ & $F+/ 918$ & $\Delta) / m$ & QNTTE & $\Delta T / F \Delta A$ & $\Delta .1 . \mathrm{r \Delta}$ & $M e / M T A$ & مشترى (انحراف معيار) \\
\hline
\end{tabular}

\section{توانبخننى}

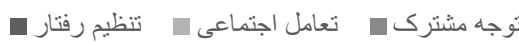

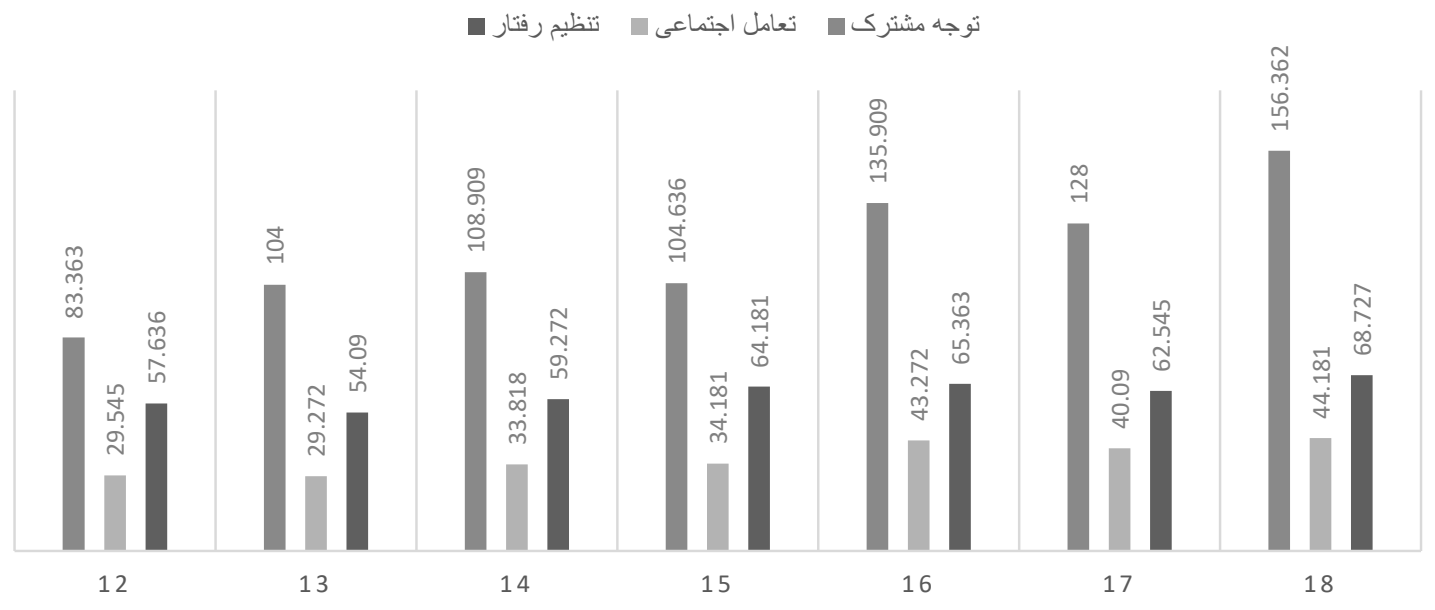


جدول r. مقايسه روند رشد كاركردهاى ارتباطى در دو ماه متوالى

\begin{tabular}{|c|c|c|c|c|}
\hline سطح معناهاري & $\mathbf{F}$ & مرجع مياتكينها & \multicolumn{2}{|c|}{ كاركردهاي ارتباطي } \\
\hline . /FAV & / $/ \Delta T Y$ & IFNTH & $\pi-\pi$ & \\
\hline$\cdot 1 \Delta \cdot 9$ &.$/ F V \Delta$ & $r 9 \Delta / 499$ & $1 F$ & \\
\hline . &.$/ 9 \%$ & $\lceil 98 / .91$ & $10-18$ & \\
\hline$\cdot / \Delta \Delta A$ &.$\%$ & $10 / / F Y$ & $18-10$ & تنظيم رفتار \\
\hline.$/ M 1$ & . /NS & AVTrat & $\mid \gamma-18$ & \\
\hline . & .1979 & 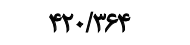 & $\mid A-I Y$ & \\
\hline . & $\%$ & $\cdot / A \mid A$ & $\mathbb{T r}-\mathbb{T}$ & \\
\hline . & $V / \Delta N G$ & $\mathrm{rrv} / \mathrm{rr}$ & $15-15$ & \\
\hline ./ATA & $\% .09$ & $1 / \% \Delta \Delta$ & $10-14$ & \\
\hline.$/ 1 \%$ & $r / M r$ & 9.9 .91 & $18-10$ & \\
\hline |grg & . Mar & $111 / \mathrm{ras}$ & $\mid \gamma-18$ & \\
\hline$+N+\Delta$ & t/Nar & $\mid A 4 / .91$ & $M-I Y$ & \\
\hline$\%+r \Lambda^{*}$ & F/AFE & $F \& A F / F \Delta \Delta$ & $\pi-i r$ & \\
\hline.$/ \% \Delta$ &.$/ 811$ & $|4 F / .9|$ & $1 f-1 r$ & \\
\hline .1999 & .1109 & $1 \mathrm{r} / / \mathrm{r}$ & $10-19$ & \\
\hline $.1 .4 \%$ & $\Delta / r \cdot r$ & 1.VAV/AMA & $18-10$ & لوجه مسترة \\
\hline צ צ. & 1.09 & $9 N \cdot 91$ & $1 Y-18$ & \\
\hline .1 .91 & $F / T$. & $M F q / F \Delta \Delta$ & $M-I V$ & \\
\hline
\end{tabular}

P $P<\cdot \Delta$

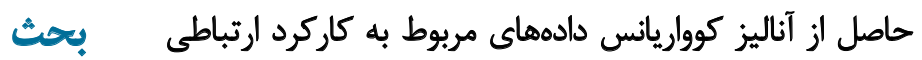

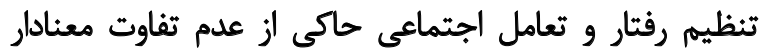

در اين مطالعه طولى از نوع مشاهدهاي، كاركردهاي ارتباطى

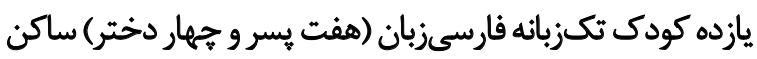
تهران در حين تعامل با مادر مورد بررسى قرار تران ترفت

نتايج حاصل از مطالعه حاكى از اين است كه ميانكين بسامد إزائ

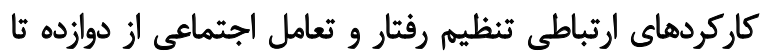

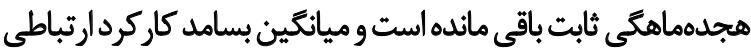

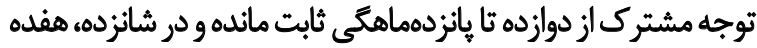

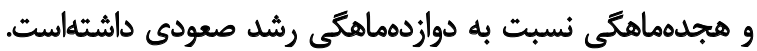

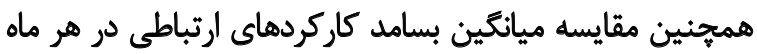

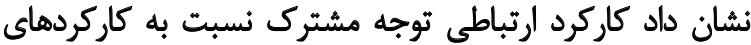

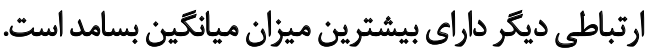
همانطور كه بيشتر ذكر شده مطالعات اندكى به روند رشد

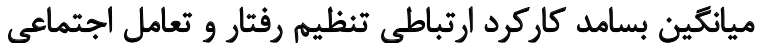

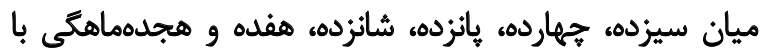

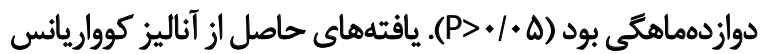

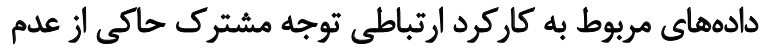

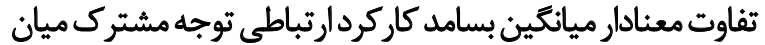

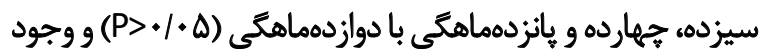

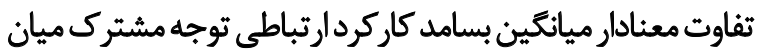

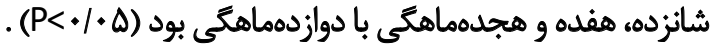
نتايج حاصل از ارزيابى و كدكذارى • r درصد از فيلمهاى

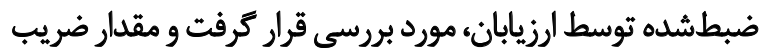

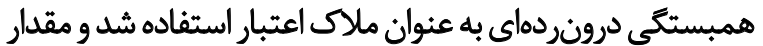
آن • • درصد به دست آمد. 
جدول "ا. مقايسه كاركردهاى ارتباطى با ماهكى با ساير ماهها

\begin{tabular}{|c|c|c|c|c|}
\hline سطح معنادارى & $\mathbf{T}$ & درجه آزادى & \multicolumn{2}{|c|}{ كاركردهاي ارتباطى } \\
\hline $.18 V 8$ & ./FTOL & W.F. & w & \multirow{6}{*}{ تنظيه رقتار } \\
\hline . ARs &.$/ T I I$ & $19 \cdot r$ & if & \\
\hline - Rear & . MIV & $\mid E M+1$ & 10 & \\
\hline.$|P \Delta|$ &.$M T$ & $10 / \Delta s$ & if & \\
\hline $.18 .$. & ./orf & $|F / F|$ & iv & \\
\hline.$/ \mathrm{VA}$ & $1 / x=$. & Wror & IA & \\
\hline.$/ 9 n$ & $.1 . H V$ & ivprese & Ir & \multirow{6}{*}{ تعامل اجتماعى } \\
\hline .18 .1 & . lart & IETM & if & \\
\hline . &.$/ 9 \pi$ & IVIOTY & 10 & \\
\hline . MIO & $1 / m \cdot r$ & Ir/TFF & 18 & \\
\hline ./MTr & V.ro & $1 \% / 94$. & iv & \\
\hline.$/ 1 Q A$ & vear & $\mid F / . P^{\circ}$ & IA & \\
\hline$\circ M$ & $1 / . r \Delta$ & $19 N \cdot \Delta$ & Ir & \multirow{6}{*}{ توجه سششرى } \\
\hline . MrV & War & IVTer & 1f & \\
\hline . Mra &.$/ 94$ & WETA & 10 & \\
\hline $.1 .19^{\circ}$ & $T / \Delta \Delta Q$ & IVATA & 18 & \\
\hline$\circ \pi$ & r/Far & 19/Arq & iv & \\
\hline $.1 \cdot \cdot r$ & r/4rqq & $1 q / / \Delta F^{\circ}$ & IA & \\
\hline
\end{tabular}

P $P<\cdot 0$

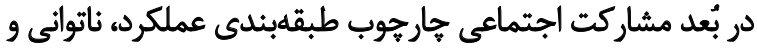

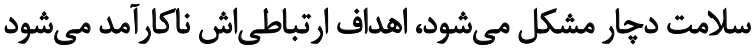

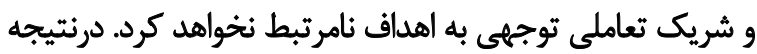

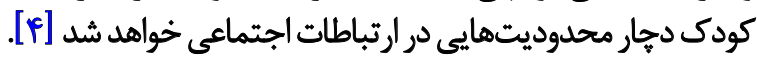
ثبات ميانكين بسامد كاركرد ارتباطى توجه مشترك از دوازده تا

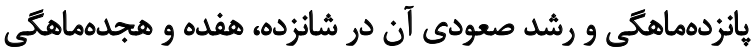

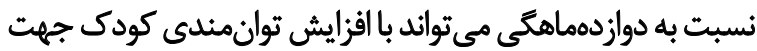

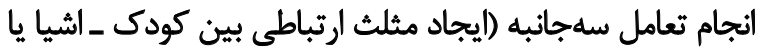

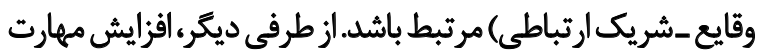

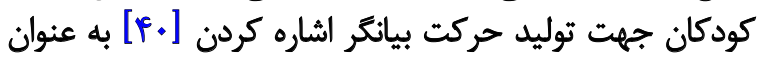

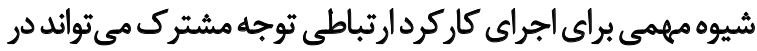

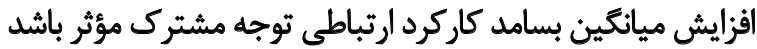

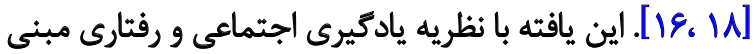

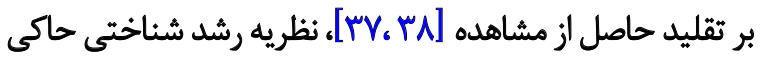

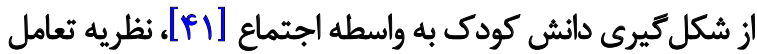

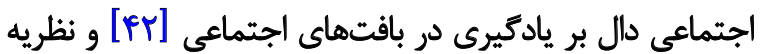

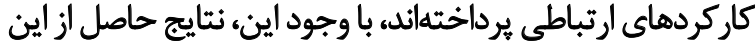

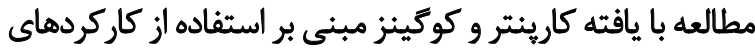

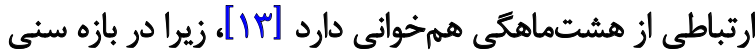

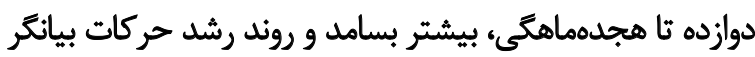

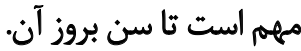
ثبات ميانكين بسامد كاركردهاى ارتباطى ثنظيم رفتار و تثعامل

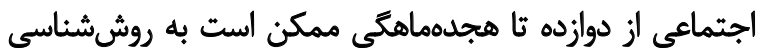

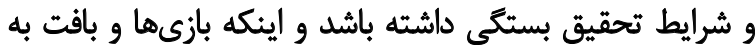

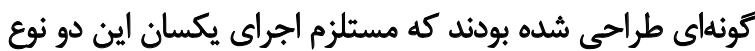

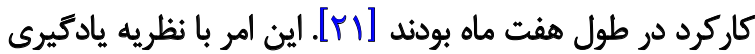

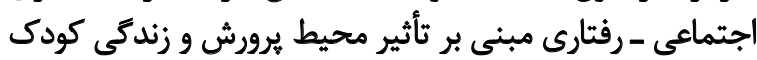

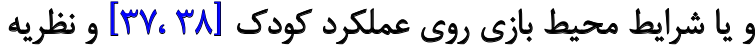

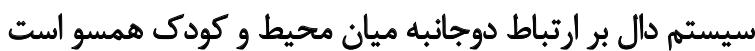

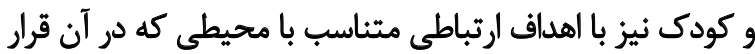

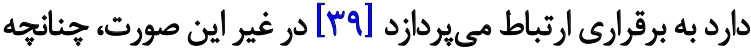

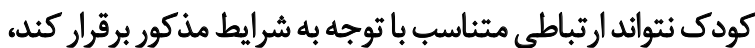


علاوه بر اين، ثرُوهش هايى در زميئه نقش كاركردهاى ارتباطى در تشخيص بهنكام مشكلات ارتباطى و زبانى صورت كيردي

ملاحظاث اخلاقى

\section{ييروى از اصول اخلاق يثوهش}

باثروهش حاضر در كميته اخلاق دانشَاه علوم بهزيستى تأييد و با كد 335.1394IR.URWR.REC ثبت شدرد

$$
\text { مامي مالى }
$$

اين مقاله بركرفته از ياياننامه كارشناسى ارشد نويسنده اول

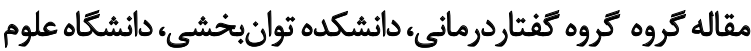

$$
\text { مشزيستى وتوانبخشى است. }
$$

مفهوم سازى، روششناسى: زهرا بابائى، طليعه ظريفيان، عطيه

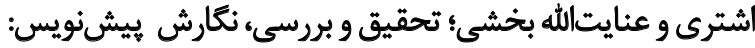

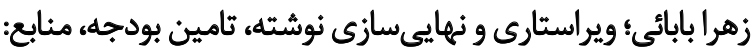

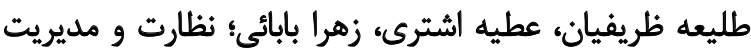

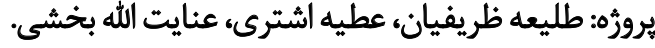

$$
\text { تعارض مثأع }
$$

نويسندكان مقاله تعارض منافعى كزارش نكردند.
سيستم [ج"] همسوست. طبق اين نظريات، كودى با مشاهده

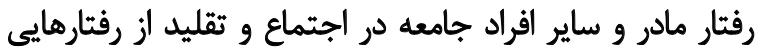

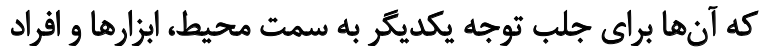

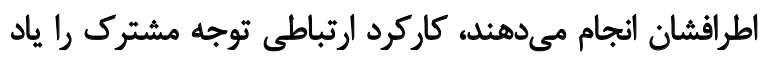
مى ميرد و از آن بيشتر استفاده مى كند. ارئر اساس نتايج حاصل از اين مطالعه ميانتين بسامد كاركردهاى

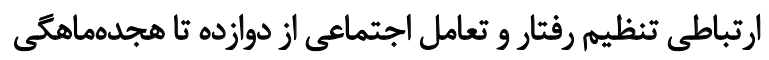

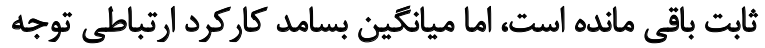

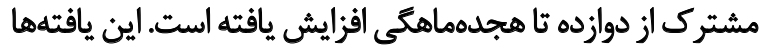

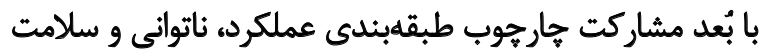

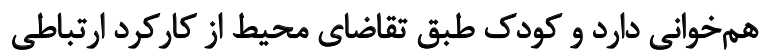

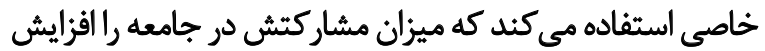

نتيجه ديگر اين مطالعه مربوط به مقايسه ميانگين بسامد إنداد

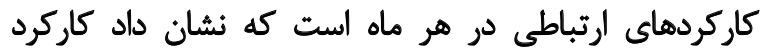

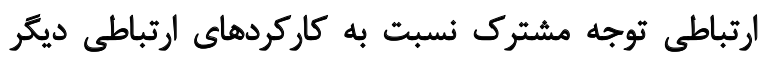

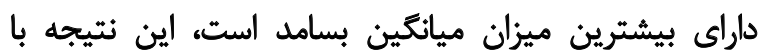

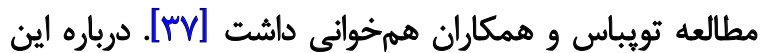

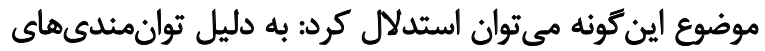

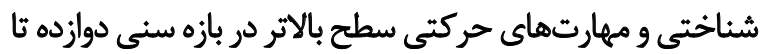

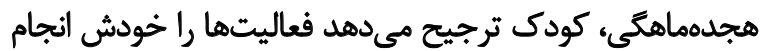

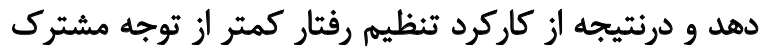

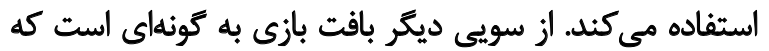

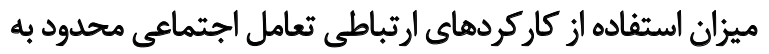

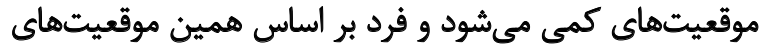

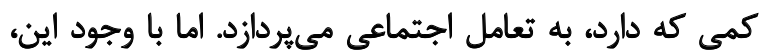

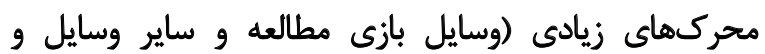

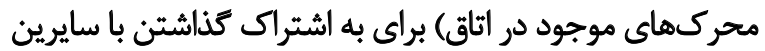

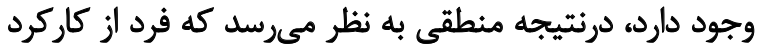
توجه مشترك بيشتر از ساير كاركردهاي ارتباطيى استفاده كندئ

\section{تنيجليَّيرى}

مطالعه حاضر نخستين مطالعه طولى است كه به تعيين روند روند

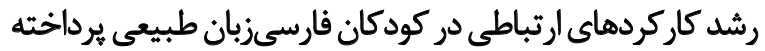

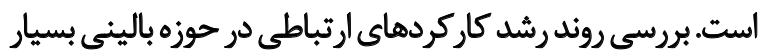

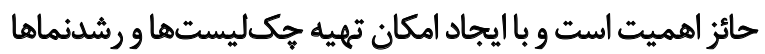

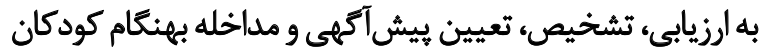
دجار اختلالات ارتباطى كمك مى كند.

حجم نمونه كم سبب مي شود در تعميم يافتههاي اين مطالعه

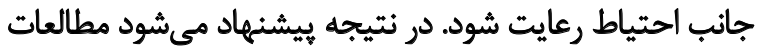

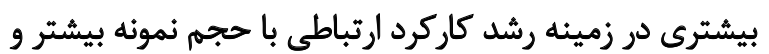

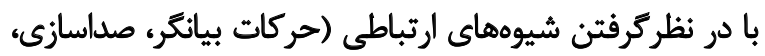

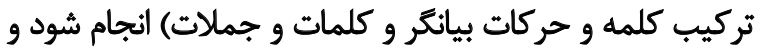




\section{References}

[1] Wetherby AM, Cain DH, Yonclas DG, Walker VG. Analysis of intentional communication of typically developing children from the prelinguistic to the multiword stage. Journal of Speech, Language, and Hearing Research. 1988; 31(2):240-52. [DOI:10.1044/jshr.3102.240] [PMID]

[2] Mitchell SJ. Infants at risk for autism spectrum disorder: Gestures in infants and mothers. [PhD. dissertation] Toronto: University of Toronto; 2013.

[3] Bates E, Benigni L, Bretherton I, Camaioni L, Volterra V. The emergence of symbols: New York: Academic Press; 1979.

[4] Bruyère SM, Van Looy SA, Peterson DB. The international classification of functioning, disability and health: Contemporary literature overview. Rehabilitation Psychology. 2005; 50(2):113-21. [DOI:10.1037/0090-5550.50.2.113]

[5] Austin J. 1962 How to do things with words. Oxford: Oxford University Press. 1962.

[6] Sax N, Weston E. Language development milestones. Edmonton: University of Alberta Press; 2007.

[7] Harding CG, Golinkoff RM. The origins of intentional vocalizations in prelinguistic infants. Child Development. 1979; 50(1):33-40. [DOI:10.2307/1129038] [PMID]

[8] Iverson JM, Goldin-Meadow S. Gesture paves the way for language development. Psychological Science. 2005; 16(5):367-71. [DOI:10.1111/ j.0956-7976.2005.01542.x] [PMID]

[9] Crais E, Douglas DD, Campbell CC. The intersection of the development of gestures and intentionality. Journal of Speech, Language, and Hearing Research. 2004; 47(3):678-94. [DOI:10.1044/10924388(2004/052)]

[10] Bruner J. The social context of language acquisition. Language \& Communication. 1981; 1(2-3):155-78. [DOI:10.1016/02715309(81)90010-0]

[11] Wetherby AM, Allen L, Cleary J, Kublin K, Goldstein H. Validity and reliability of the communication and symbolic behavior scales developmental profile with very young children. Journal of Speech, Language, and Hearing Research. 2002; 45(6):1202-18. [DOI:10.1044/10924388(2002/097)]

[12] Carpenter M, Nagell K, Tomasello M, Butterworth G, Moore C. Social cognition, joint attention, and communicative competence from 9 to 15 months of age. Monographs of the Society for Research in Child Development. 1998; 64(4):74-108. [DOI:10.2307/1166214] [PMID]

[13] Carpenter RL, Mastergeorge AM, Coggins TE. The acquisition of communicative intentions in infants eight to fifteen months of age. Language and Speech. 1983; 26(2):101-16. [DOI:10.1177/002383098 302600201] [PMID]

[14] Blake J, Osborne P, Cabral M, Gluck P. The development of communicative gestures in Japanese infants. First Language. 2003; 23(1):3-20. [DOI:10.1177/0142723703023001001]

[15] Kuhn LJ, Willoughby MT, Wilbourn MP, Vernon-Feagans L, Blair CB. Early communicative gestures prospectively predict language development and executive function in early childhood. Child Development. 2014; 85(5):1898-914. [DOI:10.1111/cdev.12249] [PMID] [PMCID]
[16] Schults A, Tulviste T, Konstabel K. Early vocabulary and gestures in Estonian children. Journal of Child Language. 2012; 39(03):664-86. [DOI:10.1017/S0305000911000225] [PMID]

[17] Jongmans MJ, Volman MCJ, Lauteslager PE. Do gestures pave the way?: A systematic review of the transitional role of gesture during the acquisition of early lexical and syntactic milestones in young children with Down syndrome. Child Language Teaching and Therapy. 2014; 31(1):71-84. [DOI:10.1177/0265659014537842]

[18] Crais ER, Watson LR, Baranek GT. Use of gesture development in profiling children's prelinguistic communication skills. American Journal of Speech-Language Pathology. 2009; 18(1):95-108. [DOI:10.1044/1058-0360(2008/07-0041)]

[19] Bates E, Thal D, Whitesell K, Fenson L, Oakes L. Integrating language and gesture in infancy. Developmental Psychology. 1989; 25(6):1004. [DOI:10.1037/0012-1649.25.6.1004]

[20] Carpendale JI, Lewis C. Constructing an understanding of mind: The development of children's social understanding within social interaction. Behavioral and Brain Sciences. 2004; 27(01):79-96. [DOI:10.1017/S0140525X04000032] [PMID]

[21] Blake J, O'Rourke P, Borzellino G. Form and function in the development of pointing and reaching gestures. Infant Behavior and Development. 1994; 17(2):195-203. [DOI:10.1016/0163-6383(94)90055-8]

[22] Capone NC, McGregor KK. Gesture developmenta review for clinical and research practices. Journal of Speech, Language, and Hearing Research. 2004; 47(1):173-86. [DOI:10.1044/1092-4388(2004/015)]

[23] Shumway S, Wetherby AM. Communicative acts of children with autism spectrum disorders in the second year of life. Journal of Speech, Language, and Hearing Research. 2009; 52(5):1139-56. [DOI:10.1044/1092-4388(2009/07-0280)]

[24] Dawson G, Toth K, Abbott R, Osterling J, Munson J, Estes A, et al. Early social attention impairments in autism: Social orienting, joint attention, and attention to distress. Developmental Psychology. 2004; 40(2):271. [DOI:10.1037/0012-1649.40.2.271] [PMID]

[25] Liszkowski U, Carpenter M, Henning A, Striano T, Tomasello M. Twelve-month-olds point to share attention and interest. Developmental Science. 2004; 7(3):297-307. [DOI:10.1111/j.14677687.2004.00349.x] [PMID]

[26] Guidetti M, Nicoladis E. Introduction to special issue: Gestures and communicative development. First Language. 2008; 28(2):107-15. [DOI:10.1177/0142723708088914]

[27] Liszkowski U. Infant pointing at twelve months: Communicative goals, motives, and social-cognitive abilities. In: Enfield, Levinson S Roots of Human Sociality: Culture, Cognition and Interaction. Oxford: Berg; 2006.

[28] Alimoradi N, Zarifian T, Ashtari A, Bakhshi E. [Development of reaching gesture and its communicative functions in 8-to 12-monthold typically developingly developing children: A longitudinal study (Persian)]. Journal of Mazandaran University of Medical Sciences. 2017; 27(152):197-202. http://jmums.mazums.ac.ir/article1-8637-fa.html

[29] Coggins TE, Carpenter RL. The communicative intention inventory: A system for observing and coding children's early intentional communication. Applied Psycholinguistics. 1981; 2(3):235-51. [DOI:10.1017/S0142716400006536] 
[30] Dale PS. Is early pragmatic development measurable? Journal of Child Language. 1980; 7(1):1-12. [DOI:10.1017/ S0305000900006991] [PMID]

[31] McShane J. Learning to talk. Cambridge: Cambridge University Press; 1980.

[32] Coggins TE, Olswang LB, Guthrie J. Assessing communicative intents in young children: Low structured observation or elicitation tasks? Journal of Speech and Hearing Disorders. 1987; 52(1):44-9. [DOI:10.1044/jshd.5201.44] [PMID]

[33] Halliday MAK. Learning how to mean--explorations in the development of language. London: Hodder Arnold; 1975. [DOI:10.1016/B978-0-12-443701-2.50025-1]

[34] Leiderman PH, Tulkin SR, Rosenfeld AH. Culture and infancy. Cambridge: Academic Press; 1977.

[35] Firuzabadi SA, Imani Jajarmi H. [Social capital and economic and social development in Tehran metropolis (Persian)]. Refahe Ejtemaei. 2006; 6(23):197-224. http://ensani.ir/file/download/ article/20101007152647

[36] Vameghi R, Sajedi F, Mojembari AK, Habiollahi A, Lornezhad HR, Delavar B. [Cross-cultural adaptation, validation and standardization of Ages and Stages Questionnaire (ASQ) in Iranian children (Persian)]. Iranian Journal Of Public Health. 2013; 42(5):522-8. [PMID] [PMCID]

[37] Bandura A, Walters RH. Social learning theory. New York: Holt Rinehart and Winston; 1977.

[38] Bronfenbrenner U. Ecology of the family as a context for human development: Research perspectives. Developmental Psychology. 1986; 22(6):723. [DOI:10.1037/0012-1649.22.6.723]

[39] Bronfenbrenner U. Ecological systems theory. London: Jessica Kingsley Publishers; 1992.

[40] Babaei Z, Zarifian T, Ashtari A, Bakhshi E, Ebrahimpour M. A look at the typically developing development of Pointing and Reaching Gestures in 12-16-Month-Old Farsi-Speaking Children: A longitudinal study. Iranian Journal of Child Neurology. 2018; 12(4):127-39. https://www.researchgate.net/publication/327801752

[41] Bandura A. Social cognitive theory: An agentic perspective. Asian Journal of Social Psychology. 1999; 2(1):21-41. [DOI:10.1111/1467-839X.00024]

[42] Vygotskij LS, Cole M. Mind in Society: The development of higher psychological process. London: England; 1978. 